\title{
Multicolor imaging in the diagnosis and follow up of type 2 acute macular neuroretinopathy
}

G De Salvo1, S Vaz-Pereira ${ }^{2,3}$, R Arora ${ }^{1,4}$ and AJ Lotery ${ }^{1,5}$

\begin{abstract}
Purpose To study the usefulness of multicolor imaging (MC) photographs in addition to near infrared reflectance (NIR) and spectral domain optical coherence tomography (SD-OCT) in the detection and follow up of acute macular neuroretinopathy (AMN).
\end{abstract}

Patients and methods Six patients with a complaint of paracentral scotomas in at least one eye due to AMN were included. They underwent full ophthalmic examination and multimodal imaging including color fundus photographs, (SD-OCT), NIR, and MC at baseline and follow up.

Results Four females and two males, aged 19-64 years, and eight eyes affected by AMN, were included. Acute phase SD-OCT in all patients confirmed the diagnosis of type 2 AMN with partial recovery of the outer retina in the convalescent phase. NIR and MC elicited in all cases hypo-reflective AMN lesions pointing toward the fovea. MC exhibited a higher contrast between the affected and the physiologic retina that slowly attenuated during the follow up showing a decrease in the hypo-reflectance of the lesions.

Conclusion MC imaging was more detailed than fundus color photographs and as detailed as NIR in the detection of AMN. When available, MC imaging should complement SD-OCT and NIR in the diagnosis and follow up of this rare inflammatory condition that may be underdiagnosed.

Eye (2017) 31, 127-131; doi:10.1038/eye.2016.193; published online 30 September 2016

\section{Introduction}

Acute macular neuroretinopathy $(\mathrm{AMN})$ is a rare retinal disorder first described by Bos and Deutman $^{1}$ in 1975. Typically, it occurs in young women complaining of a sudden decrease in visual acuity or paracentral scotoma. In such patients, they noted 'darkish brown-red, wedgeshaped dots in the macula pointing the fovea'. Advanced imaging modalities, such as spectral domain optical coherence tomography (SDOCT), have demonstrated disruption of the ellipsoid zone to be a characteristic finding in AMN suggesting to rename it acute macular outer retinopathy. ${ }^{2,3}$ Multicolor imaging (MC) and near infrared reflectance (NIR) have improved the en-face visualization of the affected areas. ${ }^{4}$ In addition, a new classification of $\mathrm{AMN}$ has been proposed ${ }^{5}$ based on the tomographic location of the lesions. Type 1 AMN, also called paracentral acute middle maculopathy, when the inner nuclear layer is involved and type 2, when the outer retina is affected (classic form). More recently, MC provides structural information originating from layers at different depths within the retina and potentially permits a more accurate definition of healthy and diseased retina. Two papers ${ }^{6,7}$ have suggested that MC imaging is more useful in the diagnosis of AMN, compared with NIR. In this report of six patients, we illustrate how MC can assist in the detection and follow up of this rare condition, in addition to NIR and SD-OCT.

\section{Subjects and methods}

Patients presented at the Medical Retina Service at the University Hospital Southampton, with a complaint of paracentral scotomas in at least one eye due to AMN. Slit lamp examination, color fundus photographs (Topcon 3D OCT 2000 or TRC 50 DX Fundus camera (Topcon, Japan)), SD-OCT, NIR, and MC (Spectralis HRA+OCT (Heidelberg Engineering, Germany)) were done. Imaging was done at the time of presentation, and repeated at 1 and/or 3 months. MC was performed at baseline and follow up in five
${ }^{1}$ Southampton Eye Unit, University Hospital Southampton Foundation Trust, Southampton, UK

${ }^{2}$ Department of Ophthalmology, Hospital de Santa Maria, Lisbon, Portugal

${ }^{3}$ Department of Ophthalmology, Faculty of Medicine, Universidade de Lisboa, Lisbon, Portugal

${ }^{4}$ Moorfields Eye Hospital, Medical retina service, National Health Service Foundation Trust, London, UK

${ }^{5}$ Department of Clinical and Experimental Sciences, Faculty of Medicine,

University of

Southampton,

Southampton, UK

Correspondence:

G De Salvo, Southampton Eye Unit, University Hospital Southampton Foundation Trust, Southampton, SO16 6YD, UK

Tel: +44 (0)23 81206442; Fax: +44 (0)23 80794120 . E-mail: gabryds|@tiscali.it

Received: 21 January 2016 Accepted in revised form: 11 July 2016 Published online: 30 September 2016 
patients, whereas only at follow up in the sixth case due to the non-availability of the MC at the time of presentation. Furthermore, the MC images underwent a manual enhancement to better highlight the contrast between the affected and the normal retina changing the Spectralis settings as follow: sharpen was set to low, whereas noise reduction was set to high.

\section{Results}

AMN was identified in four females and two males. Median age was 32.3 (range 19-64) years. Three females (Figures 1, 2, 3, and 4) had ocular symptoms few days following an episode of flu with high fever $\left(39^{\circ} \mathrm{C}\right)$, whereas one male (Figure 5) developed flu-like symptoms following

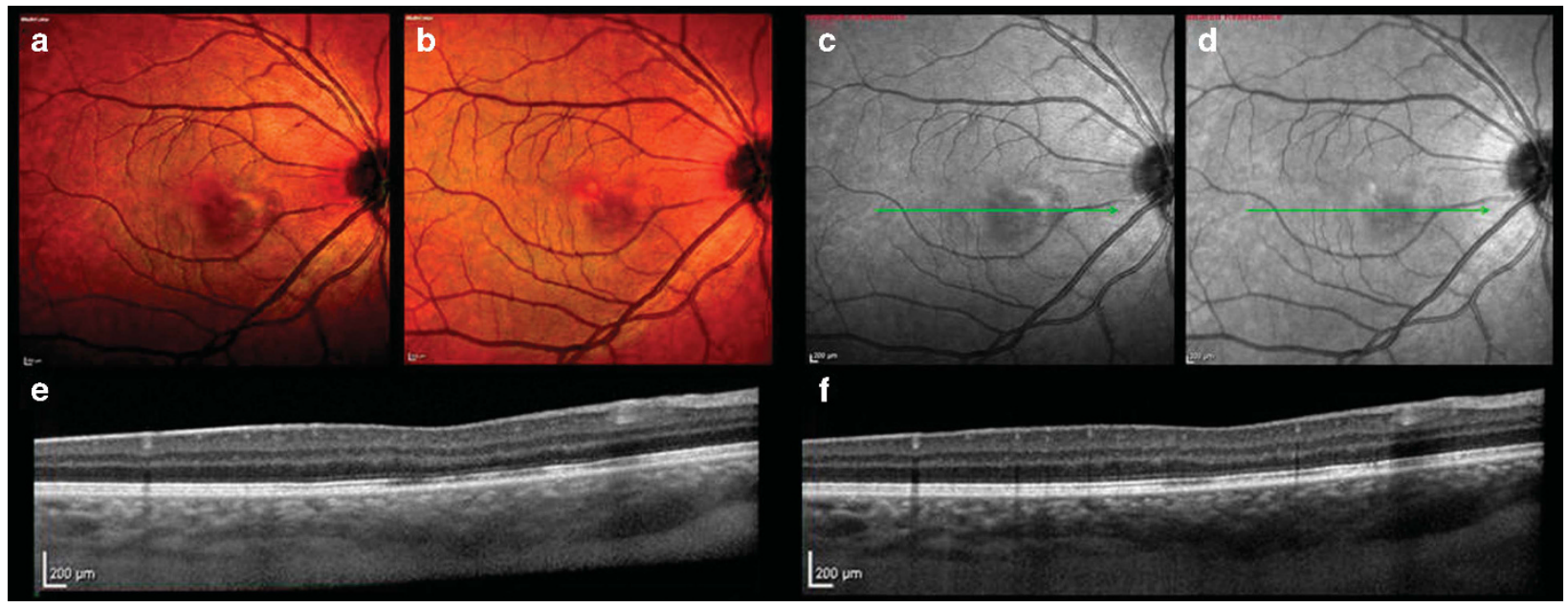

Figure 1 Patient 1, right eye: flu symptoms. (a) MC photographs of the right eye at baseline showing three dense patches of hyporeflectivity pointing toward the fovea representing the AMN lesions. (b) MC photographs of the right eye at 3 months follow up. The AMN lesions are attenuating and losing their contour and density. (c) NIR of the right eye at baseline showing the same hypo-reflective lesions seen with MC. (d) NIR of the right eye at 3 months follow up. The AMN lesions are fading off. (e) SD-OCT of the right eye shows disruption of both the ellipsoid and interdigitation zones with corresponding outer plexiform layer thickening and outer nuclear layer thinning. (f) SD-OCT of the right eye at 3 months exhibits partial restoration of the outer retina with persistent disruption of the ellipsoid and interdigitation zones.

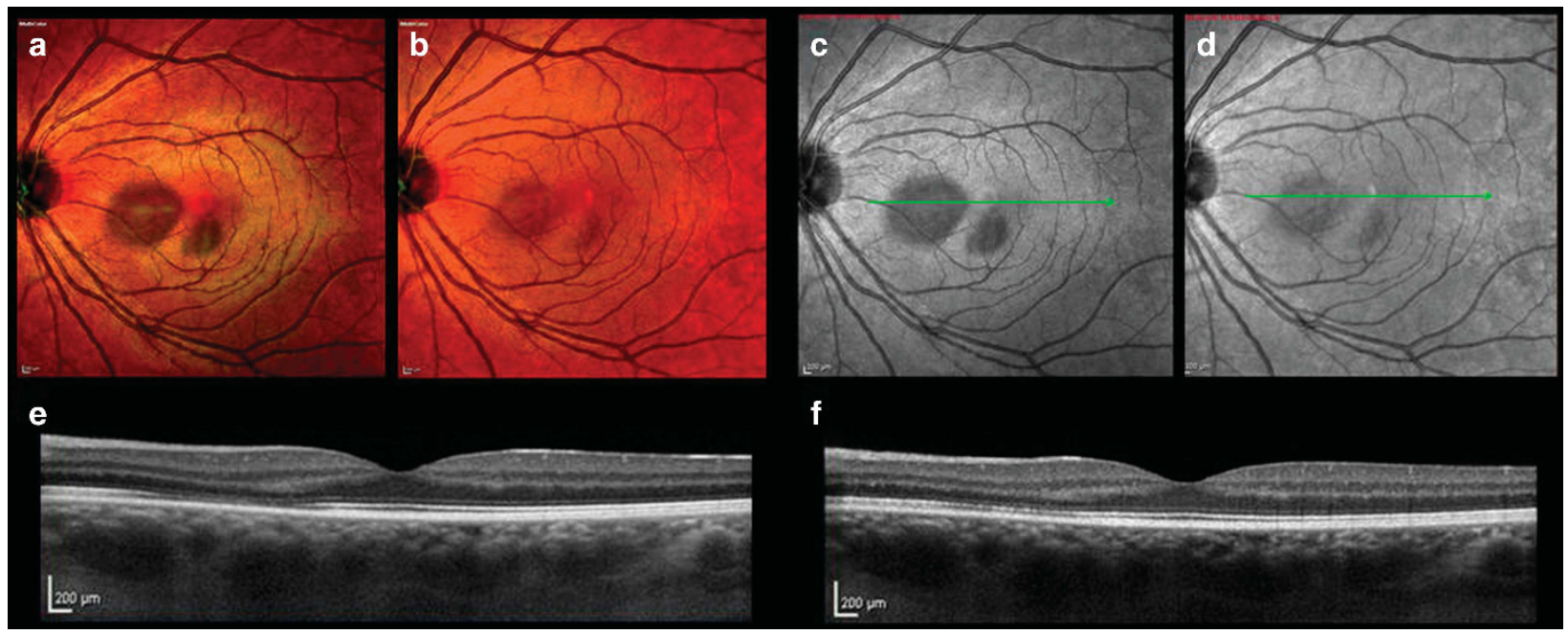

Figure 2 Patient 1, left eye: flu symptoms. (a) MC photographs of the left eye at baseline showing two dense patches of hyporeflectivity pointing toward the fovea representing AMN. (b) MC photographs of the left eye 3 months later. The AMN lesions are attenuating and losing their contour and density. (c) NIR of the left eye at baseline showing the same hypo-reflective lesions seen with MC. (d) NIR of the left eye at 3 months follow up. The AMN lesions are less defined. (e) SD-OCT of the left eye shows disruption of both the ellipsoid and interdigitation zones with corresponding outer plexiform layer thickening and outer nuclear layer thinning. (f) SD-OCT of the left eye 3 months later with partial restoration of the outer retina with persistent disruption of the ellipsoid and interdigitation zones. 
a seasonal flu vaccination. The youngest patient (Figure 6) had ocular symptoms following a trip to Thailand, where she contracted dengue fever. The latest patient of our series was another male (Figure 7) who developed traumatic retinopathy presenting as AMN following a car accident. Acute phase SD-OCT in all patients confirmed the

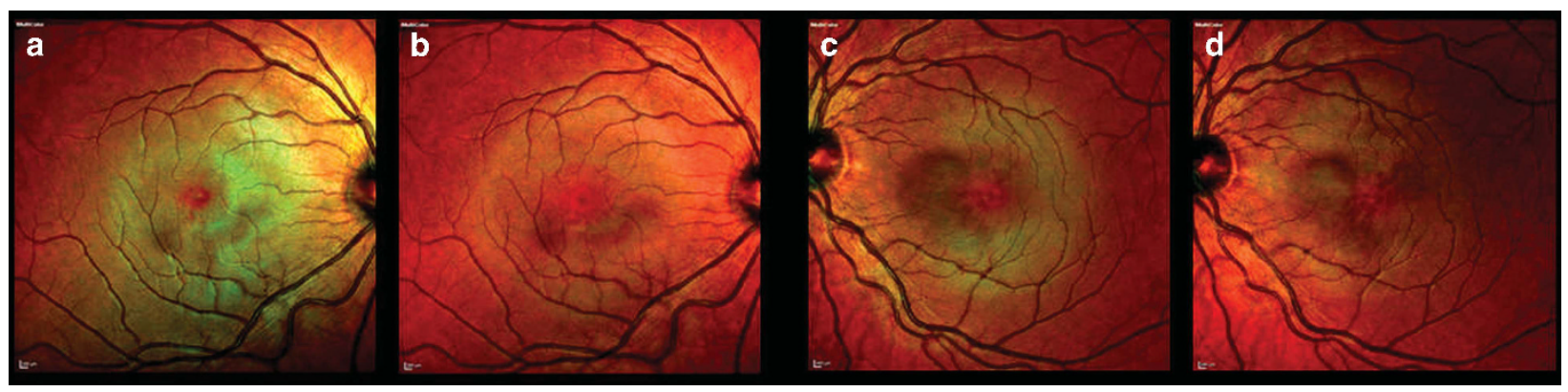

Figure 3 Patient 2: flu symptoms. (a) MC photographs of the right eye at baseline showing two patches of hypo-reflectivity surrounding the fovea representing AMN. (b) MC photographs of the right eye 3 months later. The AMN lesions are attenuating and losing their contour and density. (c) MC photographs of the left eye at baseline showing three dense patches of hypo-reflectivity pointing toward the fovea representing AMN. (d) MC photographs of the left eye 3 months later. The AMN lesions are attenuating and losing their contour and density.

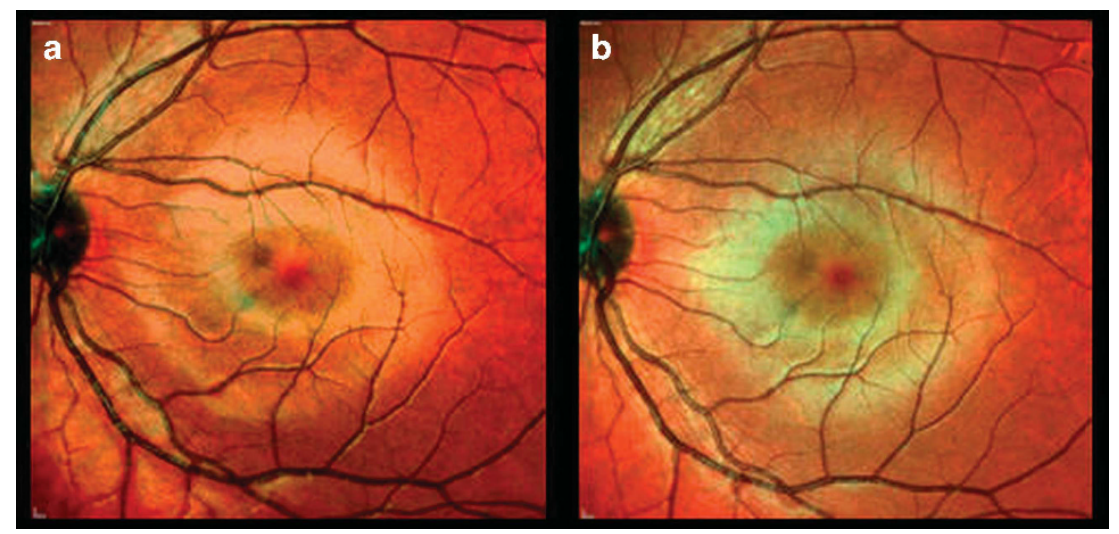

Figure 4 Patient 3: flu symptoms. (a) MC photographs of the left eye at baseline showing two rounded dense patches of hyporeflectivity representing AMN. (b) MC photographs of the left eye 1 month later. The AMN lesions are attenuating and losing their contour and density.

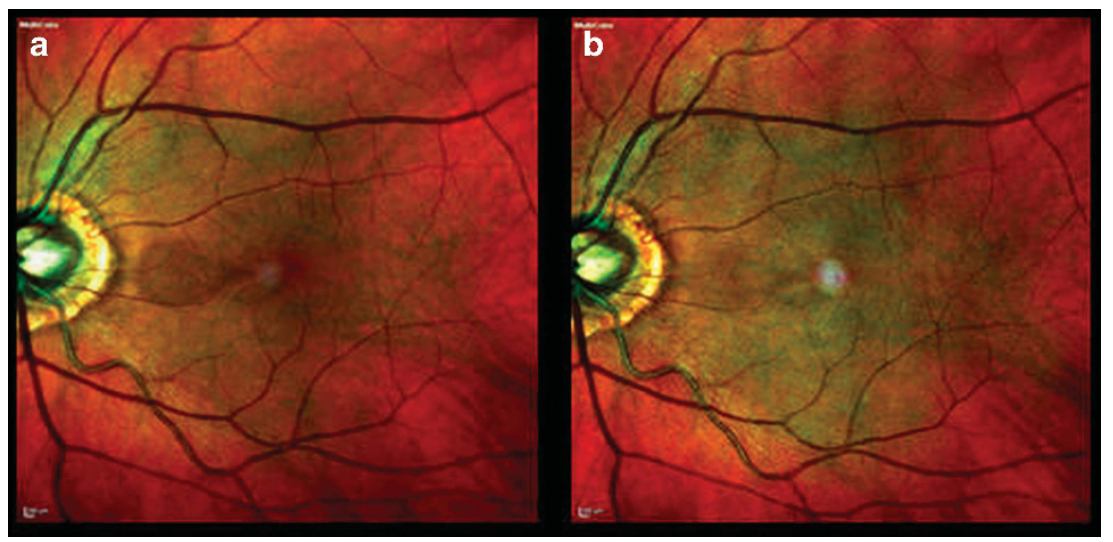

Figure 5 Patient 4: post-vaccination. (a) MC photographs of the left eye at baseline showing one hypo-reflective lesion pointing toward the fovea representing AMN. (b) MC photographs of the left eye 3 months later. No visible AMN. 
diagnosis of type 2 AMN, SD-OCT showed disruption of the ellipsoid zone, thinning of the outer nuclear layer and thickening of the outer plexiform layer. NIR and MC elicited in all cases corresponding areas of hypo-reflectance pointing toward the fovea. At MC, the lesions appeared more brownish when compared with the red of the healthy retina. Follow-up examination demonstrated improvement of symptoms in all patients but one, with gradual decrease of the hypo-reflectance of the lesions at NIR and MC, and

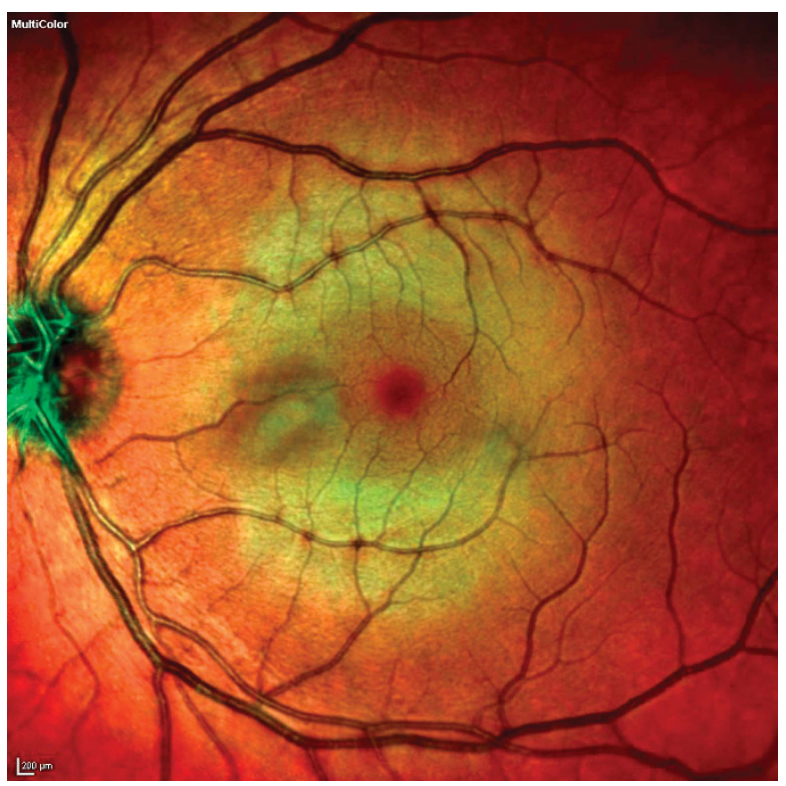

Figure 6 Patient 5: Dengue fever. MC photographs of the left eye at 3 months showing two hypo-reflective lesions representing AMN. The AMN lesion located nasally to the fovea has an annular shape with an attenuated reflectivity in the center. No baseline images are available. the latter demonstrating lower contrast between the affected and the physiological retina. All cases had partial recovery of the ellipsoid zone at SD-OCT.

\section{Discussion}

The pathogenesis of AMN remains unknown, but an underlying ischemic etiology has been suspected based on few risk factors: oral contraceptive, ${ }^{1,8}$ hypotension, ${ }^{9}$ caffeine, ${ }^{10}$ epinephrine, ${ }^{11}$ and adrenaline. ${ }^{12}$ Post-viral, retinal capillary ischemia, post-traumatic causes, ${ }^{13}$ and new associations with other systemic diseases ${ }^{14,15}$ have been recently described. Diagnosis has been classically made by fundus examination, but current multimodal imaging advances have improved AMN's detection by using both NIR and SD-OCT. Interestingly in our series, one of the patients developed AMN after a flu vaccination, which, to the best of our knowledge, has not been previously described. This may be related to the vaccination or may just be a coincidental finding. The common denominator in our case series was a viral infection, including dengue fever, previously described by others. ${ }^{15}$ Its SD-OCT findings are non-specific for the disease, but fundoscopy revealed other signs such as cotton wool spots that support the ischemic hypothesis. Furthermore, one of our patients presented with AMN features following an indirect trauma. In this latest case though a superficial retinal hemorrhage was also seen. In our study, we describe the usefulness of $\mathrm{MC}$ in the diagnosis and follow up of AMN. MC imaging was more detailed than fundus color photography and as detailed as NIR in the detection of AMN. In conclusion, AMN can be detected by a proper evaluation in conjunction with retinal multimodal imaging. This diagnostic approach is the key to suspect AMN and avoid an extensive and

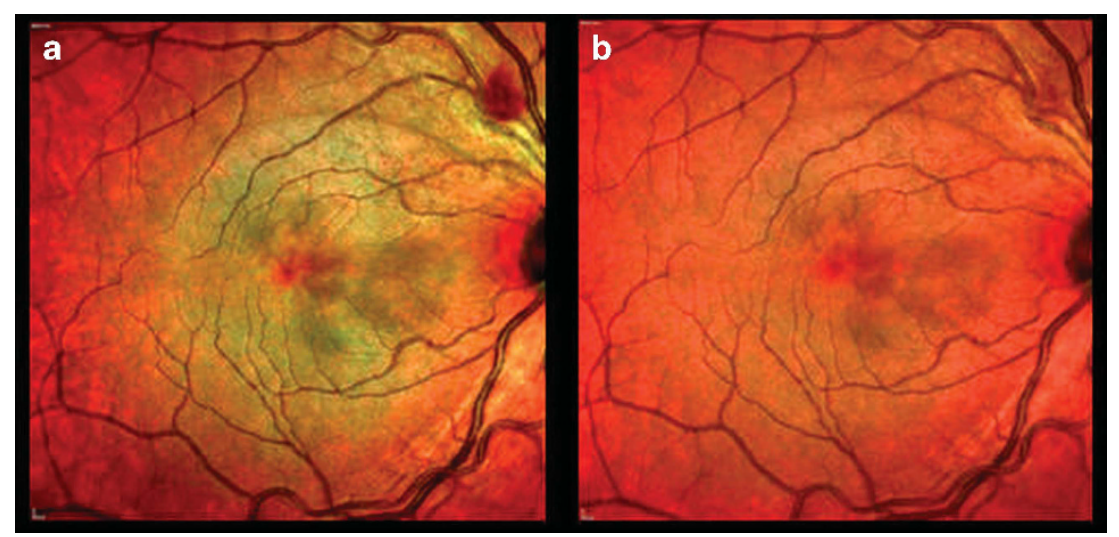

Figure 7 Patient 6: post-traumatic. (a) MC photographs of the right eye at baseline showing five dense patches of hypo-reflectivity representing AMN pointing toward the fovea. Note a superficial hemorrhage along the supra-temporal arcade. (b) MC photographs of the right eye 1 month later. The AMN lesions are attenuating and losing their contour and density. The hemorrhage along the supratemporal arcade disappeared. 
expensive work-up. When available, MC imaging should complement SD-OCT and NIR in the diagnosis and follow up of this rare inflammatory condition that may be underdiagnosed. New imaging techniques, such as OCT angiography, may provide further enlightenment on the hypothesized ischemic etiology of AMN.

\section{Summary}

What was known before

- Acute macular neuroretinopathy is a rare disease characterized by single or multiple scotomas affecting the paracentral macula in otherwise healthy patients.

- Its pathogenesis remains unknown and the classic retinal lesions are often very subtle or even absent on clinical examination making the diagnosis a real challenge for the ophthalmologists.

- Recently multicolor imaging (MC) and near infrared reflectance have improved the en-face visualization of the affected areas.

\section{What this study adds}

- With this paper, we saw that the new MC has proven its usefulness in the detection and follow up of this rare condition in a series of patients.

\section{Conflict of interest}

The authors declare no conflict of interest.

\section{References}

1 Bos PJ, Deutman AF. Acute macular neuroretinopathy. Am J Ophthalmol 1975; 80: 573-584.

2 Yeh S, Hwang TS, Weleber RG, Watzke RC, Francis PJ. Acute macular outer retinopathy (AMOR): a reappraisal of acute macular neuroretinopathy using multimodality diagnostic testing. Arch Ophthalmol 2011; 129: 365-368.
3 Hughes EH, Siow YC, Hunyor AP. Acute macular neuroretinopathy: anatomic localisation of the lesion with high-resolution OCT. Eye (Lond) 2009; 23: 2132-2134.

4 Yzer S, Freund KB, Engelbert M. Imaging in the diagnosis and management of acute macular neuroretinopathy. Int Ophthalmol Clin 2012; 52: 269-273.

5 Sarraf D, Rahimy E, Fawzi AA, Sohn E, Barbazetto I, Zacks DN et al. Paracentral acute middle maculopathy: a new variant of acute macular neuroretinopathy associated with retinal capillary ischemia. JAMA Ophthalmol 2013; 131: 1275-1287.

6 Affortit AS, Lazrak Z, Leze RH, Basdekidou C, Caputo G, Vignal C. En Face spectral domain optical coherence tomography in a case of bilateral acute macular neuroretinopathy. Retina 2015; 35: 1049-1050.

7 Capuano V, Souied EH, Semoun O, Cavallero E, Querques G. [Multicolor imaging in a case of acute macular neuroretinopathy]. J Fr Ophtalmol 2015; 38: e19-e21.

8 Miller MH, Spalton DJ, Fitzke FW, Bird AC. Acute macular neuroretinopathy. Ophthalmology 1989; 96: 265-269.

9 Leys M, Van Slycken S, Koller J, Van de Sompel W. Acute macular neuroretinopathy after shock. Bull Soc Belge Ophtalmol 1991; 241: 95-104.

10 Kerrison JB, Pollock SC, Biousse V, Newman NJ. Coffee and doughnut maculopathy: a cause of acute central ring scotomas. Br J Ophthalmol 2000; 84: 158-164.

11 Guzak SV, Kalina RE, Chenoweth RG. Acute macular neuroretinopathy following adverse reaction to intravenous contrast media. Retina 1983; 3: 312-317.

12 El-Dairi M, Bhatti MT, Vaphiades MS. A shot of adrenaline. Surv Ophthalmol 2009; 54: 618-624.

13 Nentwich MM, Leys A, Cramer A, Ulbig MW. Traumatic retinopathy presenting as acute macular neuroretinopathy. Br J Ophthalmol 2013; 97: 1268-1272.

14 Munk MR, Jampol LM, Cunha Souza E, de Andrade GC, Esmaili DD, Sarraf D et al. New associations of classic acute macular neuroretinopathy. $\mathrm{Br} J$ Ophthalmol 2015; 100: 389-394.

15 Li M, Zhang X, Ji Y, Ye B, Wen F. Acute macular neuroretinopathy in dengue fever: short-term prospectively followed up case series. JAMA Ophthalmol 2015; 133: 1329-1333. 